Communications in Physics, Vol.31, No. 1 (2021), pp. 77-83

DOI:10.15625/0868-3166/15328

\title{
PHOTOCURRENTS IN GaAs QUANTUM WIRES INCLUDING EXCITONIC EFFECTS
}

\author{
CONG NGO AND HUYNH THANH DUC ${ }^{\dagger}$
}

Ho Chi Minh City Institute of Physics, Vietnam Academy of Science and Technology, 1 Mac Dinh Chi Street, District 1, Ho Chi Minh City, Vietnam

E-mail: †htduc@hcmip.vast.vn

Received 3 August 2020

Accepted for publication 4 September 2020

Published 6 January 2021

\begin{abstract}
Photocurrent induced by ultrafast laser pulses in [001]-oriented GaAs quantum wires with a square cross section is investigated using the multiband semiconductor Bloch equations with the inclusion of electron-hole interaction. Influences of excitonic effects and quantum confinement effects on the photocurrent are evaluated and discussed.
\end{abstract}

Keywords: exciton, photocurrent, semiconductor III-V, quantum wires..

Classification numbers: 71.35.-y, 72.40.+w, 78.66.Fd, 78.67.Lt..

\section{INTRODUCTION}

Electric currents can be optically generated in noncentrosymmetric materials due to the lack of inversion symmetry in their crystal structure. Depending on the formation mechanism, photocurrents can be classified into different types: ballistic, shift, and rectification currents. These photocurrents have been investigated in bulk materials [1-14] and quantum wells (QW) [15-21] but have not received much attention in one-dimensional (1D) systems such as quantum wires (QWR). Here, we present a theoretical study of the photocurrents in semiconductor QWRs. Our method is based on solving the multiband semiconductor Bloch equations (SBE) formulated in the basis of QWR eigenfunctions. Among several models to compute the electronic band structure, we choose to use the 14-band $\mathbf{k} \cdot \mathbf{p}$ model because it is able to describe the inversion asymmetry of GaAs and can be easily applied to semiconductor heterostructures. Using this approach, we recently obtained photocurrents including excitonic effects in bulk GaAs [11] and GaAs QWs [21]. It is shown that excitonic effects not only drastically change the existing shift current but also give 
rise to a ballistic current which is absent if the electron-hole attraction is not included. Furthermore, the beats between coherently excitonic states can lead to oscillations of the ballistic current. These excitonic effects are expected to be more pronounced in semiconductor QWRs because the quantum confinement in two dimensions increases the attraction between an electron and a hole leading to greater exciton binding energy and exciton oscillator strength. Beside excitonic effects, quantum confinement effects such as valence-band mixing and the effect of symmetries determined by the crystallographic orientation and cross-sectional shape of the QWR on the generation of photocurrents are also considered in the present paper.

\section{THEORETICAL APPROACH}

To determine the electronic band structure of GaAs QWRs we employ the 14-band $\mathbf{k} \cdot \mathbf{p}$ theory in envelope function approximation [22-24]. The wave function of an electron in a QWR oriented along the $x$-axis is described by $\psi_{k}^{\lambda}(\mathbf{r})=e^{i k x} u_{k}^{\lambda}(\mathbf{r})$, where $\lambda$ is the subband index and $k$ is the $1 \mathrm{D}$ wave vector. By expressing function $u_{k}^{\lambda}(\mathbf{r})$ through the set of 14 periodic basis functions $\left\{u_{n}(\mathbf{r})\right\}$,

$$
u_{k}^{\lambda}(\mathbf{r})=\sum_{n=1}^{14} f_{n k}^{\lambda}(y, z) u_{n}(\mathbf{r})
$$

one obtains the Schrödinger equation for the slowly varying envelope function $f_{n k_{x}}^{\lambda}(y, z)$,

$$
\sum_{m=1}^{14}\left[H_{n m}^{k \cdot p}(\mathbf{k})+V_{n}^{\mathrm{conf}}(y, z) \delta_{n m}\right] f_{m k}^{\lambda}(y, z)=\varepsilon_{\lambda}(k) f_{n k}^{\lambda}(y, z) .
$$

Here, $H^{k \cdot p}(\mathbf{k})$ is the $14 \times 14 \mathbf{k} \cdot \mathbf{p}$ Hamiltonian with $\mathbf{k}=\left(k_{x}, k_{y}, k_{z}\right)$ is replaced by $\mathbf{k}=\left(k,-i \partial_{y},-i \partial_{z}\right)$, and $V_{n}^{\text {conf }}(y, z)$ are the finite band-offset potentials. We numerically solve the Eq. (2) for GaAs $/ \mathrm{Al}_{0.35} \mathrm{Ga}_{0.65} \mathrm{As} \mathrm{QWRs}$ using material papameters given in Ref. 24 . The energy dispersions of several highest valence bands and lowest conduction bands for a [001]-oriented QWR with a square cross section of $L_{y}=L_{z}=12 \mathrm{~nm}$ at room temperature $(T=300 \mathrm{~K})$ are plotted in Fig. 1 . The cross section and orientation of the QWR with respect to crystallographic directions are shown in the inset plot.

The dynamics of the photoexcited system is described by the multiband semiconductor Bloch equations [25]. By using the velocity gauge for the light-matter interaction and treating the many-body Coulomb interaction within the time-dependent Hartree-Fock approximation (HFA), we obtain the equation of motion for the density matrix in the basis consisting of QWR eigenfunctions

$$
\begin{aligned}
\frac{d}{d t} \rho_{\lambda \mu}(k, t)= & \frac{i}{\hbar}\left(\varepsilon_{\mu}(k)-\varepsilon_{\lambda}(k)\right) \rho_{\lambda \mu}(k, t)+i \sum_{v}\left[\rho_{\lambda v}(k, t) \Omega_{v \mu}(k, t)-\Omega_{\lambda v}(k, t) \rho_{v \mu}(k, t)\right] \\
& +\left(\left.\frac{d}{d t} \rho(k, t)\right|_{\text {scatt }}\right)_{\lambda \mu},
\end{aligned}
$$

where

$$
\Omega_{\lambda \mu}(k, t)=\frac{1}{\hbar}\left[\frac{e}{m_{0}} \mathbf{A}(t) \cdot \mathbf{p}_{\lambda \mu}(k)-\sum_{\lambda^{\prime}, \mu^{\prime}, k^{\prime}} V^{\lambda \lambda^{\prime} \mu^{\prime} \mu}\left(k, k^{\prime}\right) \rho_{\lambda^{\prime} \mu^{\prime}}\left(k^{\prime}, t\right)\right] .
$$




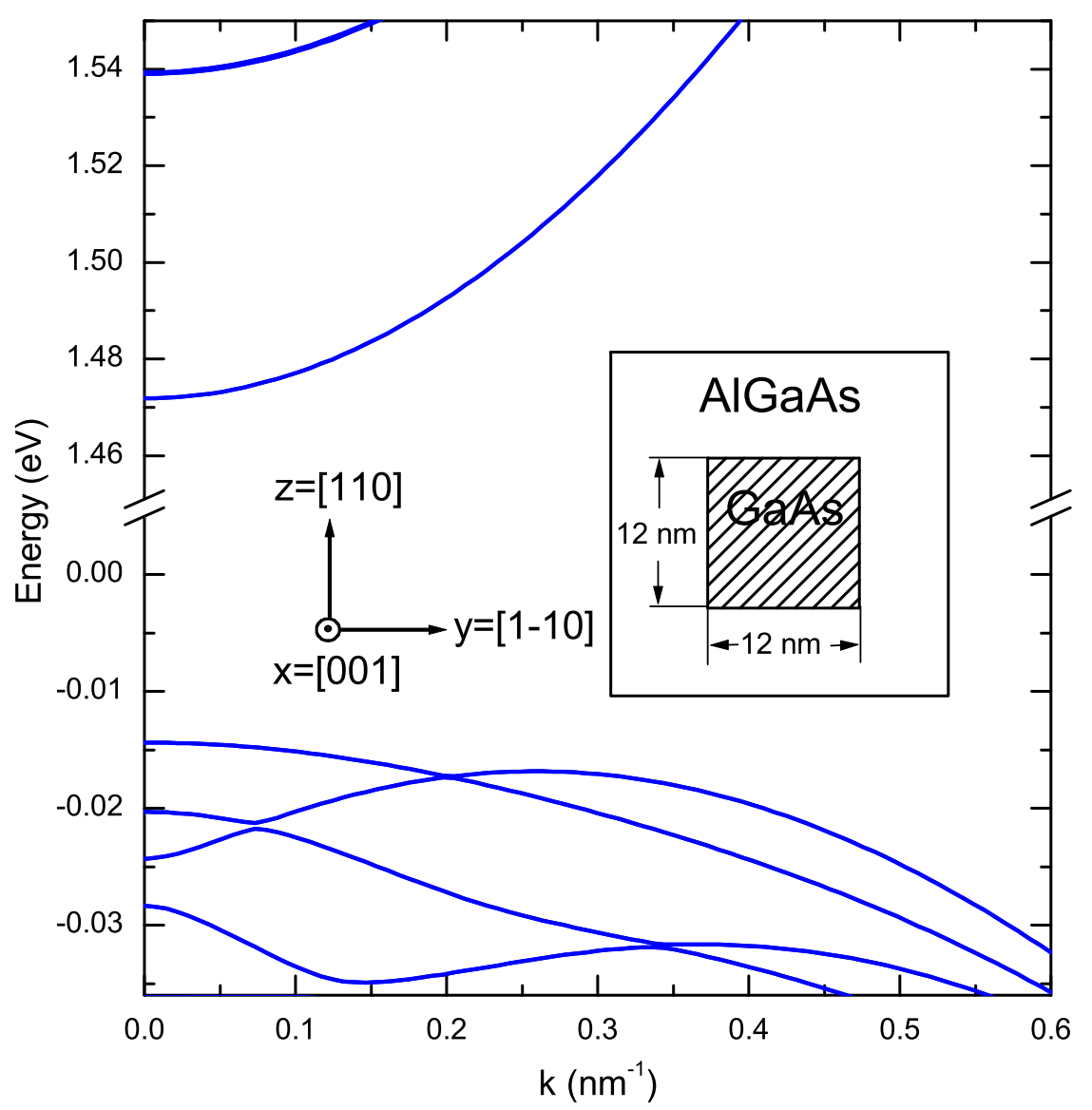

Fig. 1. Band structure of a [001]-oriented $G a A s / A l_{0.35} G a_{0.65} A s$ QWR with a square cross section. The inset shows the cross section and orientation of the QWR.

Here, $\mathbf{A}(t)$ is the vector potential of the light field and $\mathbf{p}(k)$ is the canonical momentum matrix. In our $\mathbf{k} \cdot \mathbf{p}$ model, the momentum matrix elements are defined by

$$
\mathbf{p}_{\lambda \mu}(k)=\int d y \int d z \sum_{n=1}^{14} f_{n k}^{\lambda *}(y, z)\left(\frac{m_{0}}{\hbar} \nabla_{\mathbf{k}} H^{k \cdot p}(\mathbf{k})\right)_{n m} f_{m k}^{\mu}(y, z) .
$$

The 1D Coulomb matrix elements between QWR eigenfunctions read

$$
\begin{aligned}
V^{\lambda \lambda^{\prime} \mu^{\prime} \mu}\left(k, k^{\prime}\right) & =\frac{2 e^{2}}{\epsilon_{\infty}} \int d y \int d y^{\prime} \int d z \int d z^{\prime} K_{0}\left(\left|k^{\prime}-k\right| \sqrt{\left(y^{\prime}-y\right)^{2}+\left(z^{\prime}-z\right)^{2}}\right) \\
& \times \sum_{m=1}^{14} f_{m k}^{\lambda *}(y, z) f_{m k^{\prime}}^{\lambda^{\prime}}(y, z) \sum_{n=1}^{14} f_{n k^{\prime}}^{\mu^{\prime} *}\left(y^{\prime}, z^{\prime}\right) f_{n k}^{\mu}\left(y^{\prime}, z^{\prime}\right),
\end{aligned}
$$


where $K_{0}$ is modified Bessel function of second kind. The last term in Eq. (3), $\left.\frac{d}{d t} \rho(k, t)\right|_{\text {scatt }}$, denotes the constribution from scattering processes and is approximately given by

$$
\left(\left.\frac{d}{d t} \rho(k, t)\right|_{\text {scatt }}\right)_{\lambda \mu}=\left\{\begin{array}{cc}
-\left[\rho_{\lambda \lambda}(k, t)-f^{\mathrm{FD}}\left(\varepsilon_{\lambda}(k), T\right)\right] / \tau_{1} & \lambda=\mu \\
-\rho_{\lambda \mu}(k, t) / \tau_{2} & \lambda \neq \mu
\end{array},\right.
$$

where $\tau_{1}$ and $\tau_{2}$ are phenomenological relaxation and dephasing times, respectively. $f^{\mathrm{FD}}\left(\varepsilon_{\lambda}(k), T\right)$ is the Fermi-Dirac distribution at temperature $T$. The chemical potential of this thermal distribution is chosen to yield the same density as the actual distribution. Since the scattering in one dimension is less effective than that in higher dimensions, we use long relaxation and dephasing times of $\tau_{1}=\tau_{2}=0.5 \mathrm{ps}$ in our calculations [26].

In velocity gauge, the vector potential $\mathbf{A}(t)$ is related to the electric field $\mathbf{E}(t)$ by $\mathbf{A}(t)=$ $\int_{-\infty}^{t} \mathbf{E}\left(t^{\prime}\right) d t^{\prime}$. We represent the electric field for the excitation laser pulse as

$$
\mathbf{E}(t)=E_{0} \mathbf{e}\left(e^{-t^{2} / 2 \tau_{L}^{2}} e^{i \omega t}+\text { c.c. }\right),
$$

where $E_{0}$ is the amplitude, $\mathbf{e}$ is the polarization direction, $\tau_{L}$ is the pulse duration, and $\omega$ is the frequency of light. In our numerical calculations, we use $E_{0}=5 \times 10^{3} \mathrm{~V} / \mathrm{cm}$ and $\tau_{L}=150 \mathrm{fs}$.

From the density matrix obtained by solving the multiband SBE (3), we can compute the electric current

$$
\mathbf{j}(t)=\frac{e}{m_{0}} \operatorname{Tr}[\mathbf{p} \rho]=\frac{e}{m_{0}} \sum_{\lambda, \mu, k} \mathbf{p}_{\lambda \mu}(k) \rho_{\mu \lambda}(k, t) .
$$

The diagonal (ballistic) contribution $(\lambda=\mu)$ to the total current $\mathbf{j}(t)$ can be expressed in the form

$$
\mathbf{j}^{\mathrm{d}}=e \sum_{\lambda, k} \mathbf{v}_{\lambda}(k) f_{\lambda}(k),
$$

where $\mathbf{v}_{\lambda}(k)=\hbar^{-1} \partial \varepsilon_{\lambda}(k) / \partial k$ is the group velocity and $f_{\lambda}(k)=\rho_{\lambda \lambda}(k)$ is the electron occupation. Since $\mathbf{v}_{\lambda}(k)$ is an odd function of $k$, nonvanishing ballistic current requires an asymmetric population of photoexcited carriers in $k$-space.

The off-diagonal contribution $(\lambda \neq \mu)$ to $\mathbf{j}(t)$ can also be represented as $[2,3,9]$

$$
\mathbf{j}^{\text {o.d }}=e \sum_{\lambda \neq \mu, k, k^{\prime}} \mathbf{R}_{\lambda \mu}\left(k, k^{\prime}\right) W_{\lambda \mu}\left(k, k^{\prime}\right),
$$

where $\mathbf{R}$ is the shift vector and $W$ is the electron transition rate. This term describes the shift of electron wave packet in real space during the interband transition, and hence is called the shift current.

\section{NUMERICAL RESULTS}

In the following, we present and discuss numerical results on the photocurrent in a square GaAs/AlGaAs QWR excited by linearly polarized laser pulses. The description of the QWR and its band structure is given as in Fig. 1. The direction and strength of the photocurrent depend on the excitation geometry. In the square QWR with the symmetry of $C_{4 v}$ point group, the photocurrent vanishes when the light polarization is parallel to the wire axis $x=[001]$. This kind of photocurrent however is present in lower symmetry systems such as rectangular QWRs. To maximize the current strength we choose the light polarization parallel to either $y=[1 \overline{1} 0]$ or $z=[110]$ axis. 
These two excitation geometries produce photocurrents with the same strength but flowing in opposite directions.
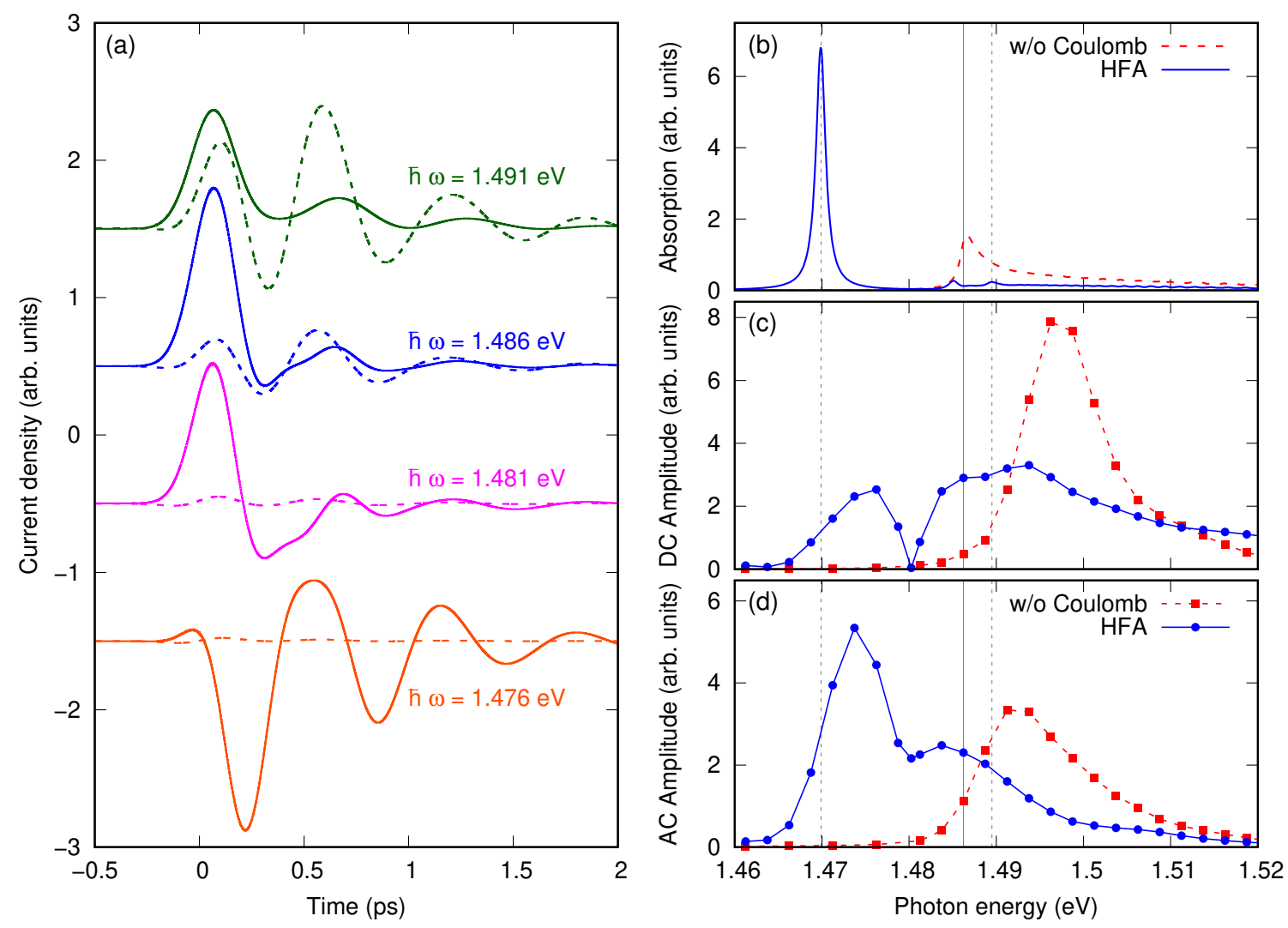

Fig. 2. (a) Time evolution of the photocurrent in the QWR for different photon energies. (b) Linear absorption spectra of the QWR. (c) DC amplitude and (d) AC amplitude of the photocurrent versus photon energy. The solid (dashed) lines are obtained by calculations with (without) Coulomb interaction. The vertical solid lines mark the bandgap energy $E_{g}=1.486 \mathrm{eV}$ and the vertical dashed lines at $1.47 \mathrm{eV}$ and $1.49 \mathrm{eV}$ highlight the heavyhole and light-hole exciton energies, respectively.

Figure 2(a) shows the time evolution of the photocurrent for different photon energies. Without Coulomb interaction, the photocurrent consists of only the shift current (see dashed lines in Fig. 2(a)). The oscillation of the shift current is due to the coherence between the heavy-hole and light-hole valence bands. When electron-hole interaction is included, besides an enhancement of the shift current, an oscillatory ballistic current arises and makes an important contribution to the total current. We note that, in noncentrosymmetric media of GaAs, Coulomb matrix elements (6) are not inversion invariant. This asymmetric Coulomb interaction between electrons and holes leads to an asymmetric occupation of photoexcited carriers in $k$-space, and thus a ballistic current.

For further analysis of the photocurrent dynamics, we perform a Fourier transformation $|j(\omega)|=|\mathscr{F}[j(t)]|$. The low-frequency spectrum of $|j(\omega)|$ basically displays two peaks. The peak at $\omega=0$ represents the direct current component while the other peak at a finite $\omega$ describes 
the alternating current component. Amplitudes of DC and AC components as a function of photon energy are shown in Figs. 2(c) and (d), respectively. Unlike the linear absorption plotted in Fig. 2(b), which follows the law $\left(\hbar \omega-E_{g}\right)^{-1 / 2}$ of the 1D density of states (see the red dashed line) and shows peaks at exciton energies (see the blue solid line), the dependence of photocurrent amplitude on photon energy is more complex. Without Coulomb interaction, the variation of photocurrent amplitudes when photon energy increases does not well reflect the 1D density of states. In particular, the amplitude does not reach the maximum at $\hbar \omega=E_{g}$ as in the case of absorption, but does at a greater photon energy. As demonstrated in Ref. [20], this difference is due to the stronger influence of mixing between heavy- and light-hole valence bands on the photocurrent than the absorption. With electron-hole attraction, due to the excitonic enhancement of absorption, the current amplitude is strongly enhanced for excitations near the bandgap. Regarding the photocurrent spectrum, we obtain a spectral shift to lower energies corresponding to the exciton binding energy. Moreover, in between the heavy-hole and light-hole exciton energies, we find an interesting zero crossing at $\hbar \omega=1.48 \mathrm{eV}$ (see Fig. 2(c)) that corresponds to a directional reversal of the photocurrent.

\section{CONCLUSIONS}

We have investigated the photocurrents induced by linearly-polarized laser pulses in GaAs QWRs. We show that the existence of ballistic current is entirely due to asymmetric electron-hole scatterings. The formation of bound excitons strongly enhances the strength of ballistic and shift currents for excitations slightly below the bandgap and causes oscillations in the transient of these currents. Together with excitonic effects, the valence-band mixing influences the photocurrent resulting in a complex dependence of current amplitudes on the excitation photon energy.

\section{ACKNOWLEDGMENT}

This work is funded by Vietnam National Foundation for Science and Technology Development (NAFOSTED) under Grant No. 103.01-2017.42. We thank the Center for Informatics and Computing, Vietnam Academy of Science and Technology for providing computing time.

\section{REFERENCES}

[1] V. I. Belinicher and B. I. Sturman, Sov. Phys. Usp. 23 (1980) 199.

[2] V. I. Belinicher, E. L. Ivchenko, and B. I. Sturman, Zh. Eksp. Teor. Fiz. 83 (1982) 649.

[3] B. I. Sturman and V. M. Fridkin, The photovoltaic and photorefractive effects in noncentrosymmetric materials, Gordon and Breach, Philadelphia, 1992.

[4] J. B. Khurgin, J. Opt. Soc. Am. B 11 (1994) 2492.

[5] J. E. Sipe and A. I. Shkrebtii, Phys. Rev. B 61 (2000) 5337.

[6] V. I. Shelest and M. V. Éntin, Sov. Phys. Semicond. 13 (1979) 1353.

[7] P. Král, J. Phys.: Condens. Matter. 12 (2000) 4851.

[8] F. Nastos and J. E. Sipe, Phys. Rev. B 74 (2006) 035201.

[9] L. E. Golub and E. L. Ivchenko, J. Exp. Theor. Phys. 112 (2011) 152-159.

[10] L. Z. Tan, F. Zheng, S. M. Young, F. Wang, S. Liu, and A. M. Rappe, npj Comput. Mater. 2 (2016) 16026.

[11] R. Podzimski, H. T. Duc, and T. Meier, Phys. Rev. B 96 (2017) 205201.

[12] A. Ghalgaoui, K. Reimann, M. Woerner, T. Elsaesser, C. Flytzanis, and K. Biermann, Phys. Rev. Lett. 121 (2018) 266602.

[13] A. M. Burger, R. Agarwal, A. Aprelev, E. Schruba, A. Gutierrez-Perez, V. M. Fridkin, and J. E. Spanier, Sci Adv. 5 (2019) eaau5588. 
[14] B. I. Sturman, Phys.-Usp. 63 (2020) 407.

[15] N. Laman, M. Bieler, and H. M. van Driel, J. Appl. Phys. 98 (2005) 103507.

[16] M. Bieler, K. Pierz, and U. Siegner, J. Appl. Phys. 100 (2006) 083710.

[17] M. Bieler, K. Pierz, U. Siegner, and P. Dawson, Phys. Rev. B 76 (2007) 161304.

[18] S. Priyadarshi, M. Leidinger, K. Pierz, A. M. Racu, U. Siegner, M. Bieler, and P. Dawson, Appl. Phys. Lett. 95 (2009) 151110.

[19] S. Priyadarshi, K. Pierz, U. Siegner, P. Dawson, and M. Bieler, Phys. Rev. B 83 (2011) 121307(R).

[20] H. T. Duc, R. Podzimski, S. Priyadarshi, M. Bieler, and T. Meier, Phys. Rev. B 94 (2016) 085305.

[21] H. T. Duc, C. Ngo, and T. Meier, Phys. Rev. B 100 (2019) 045308.

[22] H. Mayer and U. Rössler, Phys. Rev. B 44 (1991) 9048.

[23] P. Pfeffer and W. Zawadzki, Phys. Rev. B 41 (1990) 1561.

[24] R. Winkler, Spin-orbit Coupling Effects in Two-Dimensional Electron and Hole Systems, Springer, Berlin, 2003.

[25] H. Haug and S. W. Koch, Quantum Theory of the Optical and Electronic Properties of Semiconductors, 4th ed., World Scientific, Singapore, 2004.

[26] H. T. Duc, Q. T. Vu, T. Meier, H. Haug, and S. W. Koch, Phys. Rev. B 74 (2006) 165328. 